\title{
A Tolerance Analysis Approach Based on Robot Kinematics
}

\author{
Xiuheng Zhang ${ }^{1,2, ~ a, ~ H o n g y i ~ L i u ~}{ }^{1, b}$ and Zhong Luo ${ }^{2, c}$ \\ ${ }^{1}$ School of Mechanical Engineering and Automation Northeastern University, Shenyang 110004, \\ China \\ ${ }^{2}$ School of Mechanical Engineeloop, Shenyang Ligong University, Shenyang 110159, China \\ aaeh25@126.com, ${ }^{b}$ liu140845@yahoo.com.cn, ${ }^{c}$ zhluo@mail.neu.edu.cn
}

Keywords: robot kinematics, Small displacement torsor, 3D tolerance model, tolerance analysis

\begin{abstract}
This paper presents a tolerance modeling approach based on robot kinematics theory within $\mathrm{CAD} / \mathrm{CAM}$ system. Based on the differential kinematics theories, each feature of characteristics including size tolerance and geometrical tolerance was established as a corresponding small displacement torsor. In order to express the relation between the small displacement of all functional features and the functional requirement, the final expression was represented to establish the projection of analysis of the tolerance chain. A mathematical expression was obtained by the mean instead of single points, and the constraints can be represented by interval using the standard deviation, not limit deviations. After having identified the unit components and the functional requirement from the tolerance chain, we obtained the percentage contribution of each unit feature to the functional requirement. A percentage contribution can help designer to decide which tolerance is tighten or loosen. The application of the tolerance analysis approach in a simple three-dimensional sample was also discussed in this paper.
\end{abstract}

\section{Introduction}

Tolerance analysis is a process that establish expressions between the given tolerances and assembly tolerance and analyze the reasonableness of the known tolerance on current assemble condition. After the design requirements were met, the accuracy of the machine will be improved by further analyze the sensitivity of functional elements relative to the total assembly tolerance without increasing the cost of production. It is a helpful method to predict the cumulative error at the design stage. And it is also a necessary step in design and manufacture of high-quality products.

A lot of work has been carried out in the field of three-dimensional tolerance analysis. A. Desrochers used matrix to models 3D tolerance zones with a variety of possible shape; E.E Lin generalized the modeling methods about cumulative tolerance in $1 \mathrm{D}, 2 \mathrm{D}$ and $3 \mathrm{D}$ through the analysis of tolerance shape; $\mathrm{Hu}$ Jie propose the general kinematics model that define the cumulative three-dimensional tolerance based on robot kinematics analysis; Massimiliano Marziale had done an objective and complete comparison among the vector loop method and matrix method, analyzing the advantages and the weakness and furnishing a criterion for their choice and application. With the mathematical view, these methods were proposed without taking into account the actual tolerance distrbution. This paper focus on similarities between the calculation of assembly dimension chain and robot kinematics. Based on differential kinematics theory, small displacement torsor tolerance model is improved. By determined with actual processing information of the functional elements, the model more accordant to practice. 


\section{D tolerance model}

The first step of tolerance analysis deals with the establishment tolerance model. We usually allocate the total assembly tolerance by dimension chain. And dimension chain composes of unit loops that represent the sizes of functional elements and close loop that represents assembly requirements. Based on the similarities between dimension chains in tolerance analysis and kinematics chain in robotics, each functional element in tolerance models is treated as the link of a robot. The nominal size of functional element, the combination between two functional elements and their tolerance zone can be considered as the nominal length of link, joint and variations in Robotics. We also assume that the functional surfaces in structures are perfect. Geometric characteristics of the surfaces can be represented with the direction and displacement of features. The tolerances are considered as the variations that determined by the change of features. Relationship between assembly requirements with functional elements is shown as Figure 1. In figure 1, part1, part2, part3 ... ..., partn are functional elements (tolerance characteristics) that affect assembly requirements, that is, unit loops; $\mathrm{T}_{\mathrm{E} 1}, \mathrm{~T}_{\mathrm{E} 2}, \mathrm{~T}_{\mathrm{E} 3}, \ldots \ldots, \mathrm{T}_{\mathrm{En}-1}, \mathrm{~T}_{\mathrm{En}}$ are tolerance values of functional elements represented with small displacement torsors; $\mathrm{T}_{\mathrm{AR}}$ is total assembly requirements, the closed component.

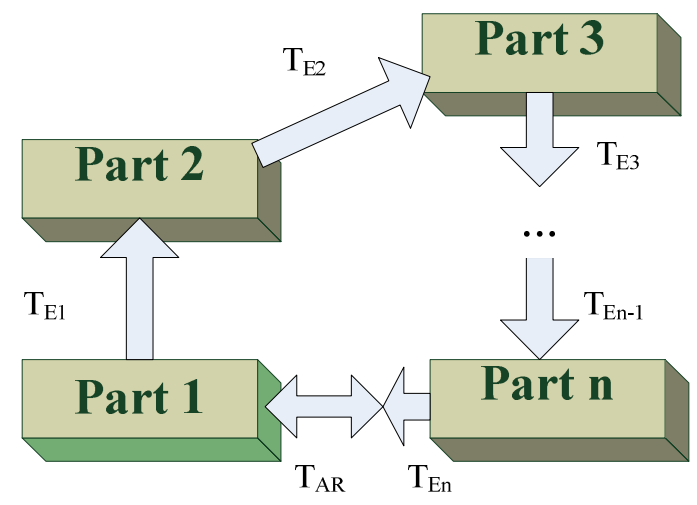

Fig. 1. The diagram of tolerance chain

A tolerance zone model representation of functional element. Based on coordinate transformation theory in Differential kinematics of robotics, the tolerances of functional elements can be expressed as:

$$
T_{E i}=\left[\begin{array}{cccc}
\mathbf{1} & -\delta_{z} & \delta_{y} & d x \\
\delta_{z} & \mathbf{1} & -\delta_{x} & d y \\
-\delta_{y} & \delta_{x} & \mathbf{1} & d z \\
\mathbf{0} & \mathbf{0} & \mathbf{0} & \mathbf{1}
\end{array}\right]
$$

where, $\delta \mathrm{x}, \delta \mathrm{y}, \delta \mathrm{z}$ - rotating components projected on the local frame;

$\mathrm{dx}, \mathrm{dy}, \mathrm{dz}$ - translation components projected on the local frame;

The small displacement torsor (SDT) can be used directly in its form [n]:

$$
T_{E i}=\left[\begin{array}{l}
d \\
\delta
\end{array}\right]=\left[\begin{array}{llllll}
d_{x} & d_{y} & d_{z} & \delta_{x} & \delta_{y} & \delta_{z}
\end{array}\right]^{T}
$$

where, $\mathrm{d}$ represents the translation dispersions, $\delta$ represents the rotation dispersions.

Since tolerance zones usually attached to the characteristics surface, tolerance zones and regular surface characteristics are known to be similar in directions, resulting in rotational and translational invariance on contact surfaces in a machine. It has some constraints between torsor 
parameters and tolerances interval. According to the definition about size and geometric tolerances, tolerance zones have nine different shapes. A. Desrochers [A. Desrochers et. al.2003] summarized their expression by the small displacement torsor method.

Cumulative modeling of 3D tolerance zones. From the view of the homogeneous transformation theory in robotics, the tolerance chains were placed in Cartesian coordinate system. The base coordinate system is located in the center of the closed-loop. And local coordinate systems of functional elements of the tolerance chain were established. Assume that the origin of the coordinate system stands in the center of tolerance zones, and project 3D-dimensional tolerances from the local coordinate system to the base coordinate system by the homogeneous transform, shown in Figure 2.

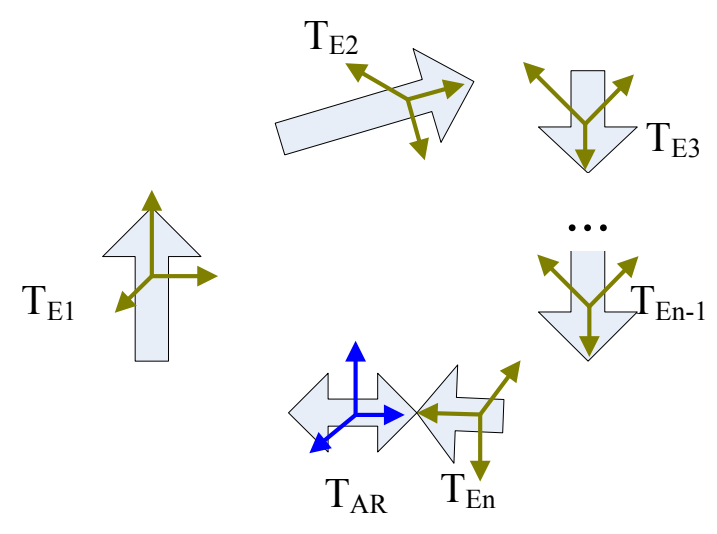

Fig. 2. The coordinate frame of tolerance chain

By robot Differential kinematics theory, each functional element transformed from the local coordinate transformation to the base coordinate system can be described as:

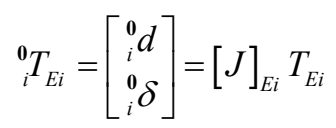

where, $[\mathrm{J}]_{E i}$ : Jacobian matrix expressing transfer function of each functional elements;

$$
[J]_{E i}=\left[\begin{array}{cc}
R^{T} & -R^{T} S(p) \\
\mathbf{0} & R^{T}
\end{array}\right]
$$

where, $[R]_{3 \times 3}$ is the rotation matrix expressing the relationship between reference coordinate system relative to the base coordinate system. $S(p)$ is a skew-symmetric matrix of the position vector $\boldsymbol{p}=\left[p_{x}, p_{;}, p_{x}\right]^{r}$, defined as:

$$
S(p)=\left[\begin{array}{ccc}
0 & -p_{z} & p_{y} \\
p_{z} & 0 & -p_{x} \\
-p_{y} & p_{x} & 0
\end{array}\right]
$$

According to probability theory, the actual size of partial components can be considered to be as a random variable that has various forms of distribution in the tolerance band. When the process is stable and mass production, distribution of the actual size is nearing the normal distribution, and the closed component tends to be normal distribution. When the distributions of partial components are non-normal distribution, the distribution coefficient $\mathrm{K}$ should be introduced to express the distribution characteristics. Tolerance of closed component is written unified as: 


$$
T_{a}=\sqrt{\sum_{i=1}^{n} K^{2}[J]_{E i}^{2} T_{E i}^{2}} \leq T_{A R}
$$

where, $\mathrm{K}$ is distribution coefficient which may take different values with different distribution, and $\mathrm{K}=1$ with normal distribution;

$T_{a}$ is the accumulated tolerance of the functional elements, which must be less than or equal to the value of the total assembly tolerance $T_{A R}$ determined by the functional requirements. To meet functional requirements, tolerance values are validated and adjusted in the design phase. Tolerance analysis can be summarized through a four steps procedure:

1. Simplify the assembly structure; identify the functional elements which contribute to the functional requirements; establish a tolerance chain including dimensional and geometric tolerance;

2. Establish the coordinate systems for partial components and closed component in tolerance chain;

3. Use Jacobian matrix to express the relative position and orientation of each component in the closed chain;

4. Determine the distribution of the partial components to calculate the cumulative tolerances.

\section{Numerical Example}

The following section will show how the four steps of the tolerance analysis methodology can be applied successfully to a simple 3D example. It is the centering pin mechanism shown in Fig.3. In this figure, some key sizes and tolerances have given to make the axial of centering pin vertical to plane K, with a precision of $\pm \mathbf{0} .1 \mathrm{~mm}$.

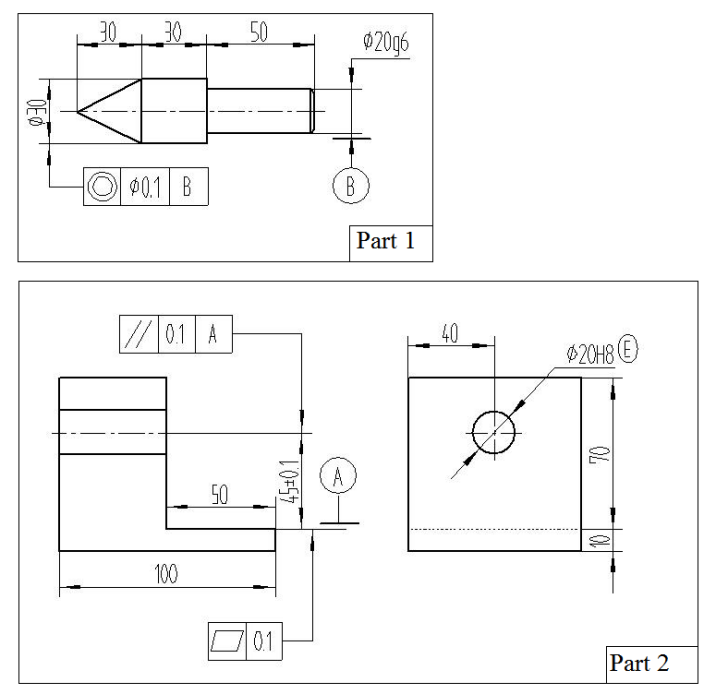

Fig.3. Parts drawing of centering pin mechanism

Description of the Example. According to this method, firstly, identify the partial components (functional elements) which had a great influence on assembly requirements. Simplify functional elements to determine tolerance chain. Geometric tolerances can be as space dimensional partial components whose basic sizes equal to NULL. So tolerance chain was established. Plane K was considered as base coordinate system and the local coordinate system lay in the center of tolerance zone of functional elements. In this case, for there is no statistical data for reference, size tolerance and geometric tolerance of partial components are regarded as triangular distribution. $\mathrm{K}=1.22$. 
Construction and Analysis of Tolerance Chain. The figure 4 shows that there are two parts involved in this case. Simplify the tolerance chain graph by identifying the functional elements.

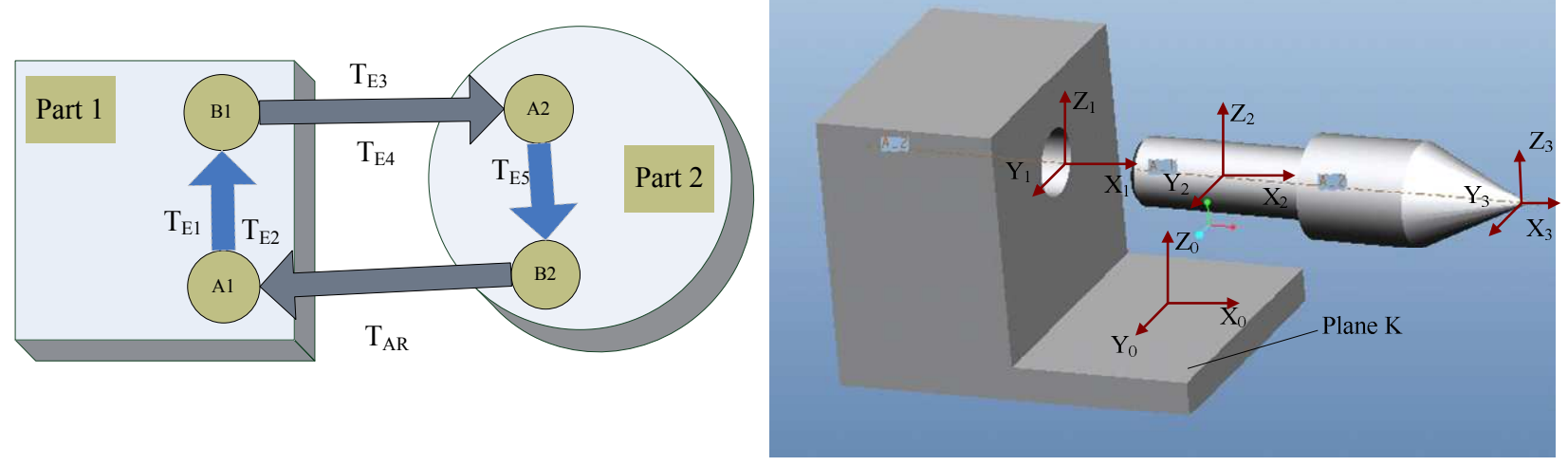

Fig.4. Kinematic chain identification

The analysis of the figure shows that, there are three shapes of the tolerance zones in this case within two parallel straight lines projected in a plane (such as dimensional tolerances); or between two parallel planes (such as flatness and parallelism); or within cylindrical space (such as coaxial). according to the literature, the torsor representation of the above tolerances was obtained by the literature[1], as shown in Table 1:

Table 1 Description of characteristic analysis of the functional elements

\begin{tabular}{|c|c|c|c|c|c|}
\hline parts & $\begin{array}{l}\text { Functional } \\
\text { elements }\end{array}$ & $\begin{array}{c}\text { Tolerance } \\
\text { characteristics }\end{array}$ & $\begin{array}{l}\text { The torsor } \\
\text { matrix of } \\
\text { tolerance } \\
\text { zones }\end{array}$ & Constraint parameters & Distribution \\
\hline \multirow{3}{*}{ Part 1} & A1 & \begin{tabular}{l|l|l|}
$T_{E 1} \quad \square$ & 0.1 \\
\end{tabular} & \multirow{2}{*}[\begin{array}{cc}{\mathbf{0}}&{\delta_{x}}\\
{\mathbf{0}}&{\delta_{y}}\\
{d_{z}}&{\mathbf{0}}\end{array}]{} & \multirow{2}{*}{$\begin{aligned}-\frac{t}{2} & \leq d_{z} \leq \frac{t}{\mathbf{2}} \\
-\frac{t}{L_{x}} & \leq \delta_{x} \leq \frac{t}{L_{x}} ;-\frac{t}{L_{y}} \leq \delta_{y} \leq \frac{t}{L_{y}}\end{aligned}$} & \multirow{2}{*}{$\begin{array}{l}\text { Triangular } \\
\text { distribution }\end{array}$} \\
\hline & $\mathrm{A} 1-\mathrm{B} 1$ & $\begin{array}{lllll}T_{E 2} & / / & 0.1 \\
\end{array}$ & & & \\
\hline & B1 & $T_{E 3} \quad \mathbf{4 5} \pm \mathbf{0 . 1}$ & \multirow{3}{*}[\begin{array}{cc}{\mathbf{0}}&{\delta_{x}}\\
{\mathbf{0}}&{\mathbf{0}}\\
{d_{z}}&{\mathbf{0}}\end{array}]{} & \multirow{3}{*}{$-\frac{t}{\mathbf{2}} \leq d_{z} \leq \frac{t}{\mathbf{2}} ;-\frac{t}{L_{x}} \leq \delta_{x} \leq \frac{t}{L_{x}}$} & \multirow{3}{*}{$\begin{array}{l}\text { Triangular } \\
\text { distribution }\end{array}$} \\
\hline \multirow{2}{*}{$\begin{array}{c}\text { Part } \\
\text { 1-Part } \\
2\end{array}$} & \multirow[t]{2}{*}{$\mathrm{B} 1-\mathrm{A} 2$} & $\begin{array}{c}T_{E 4} \\
\left.\phi \mathbf{2 0} H \mathbf{8}_{0}^{+0.033}\right)\end{array}$ & & & \\
\hline & & $T_{E 5} \quad \phi 20 g 6\left({ }_{-0.016}^{-0.007}\right)$ & & & \\
\hline Part 2 & $\mathrm{~A} 2-\mathrm{B} 2$ & \begin{tabular}{l|l|ll|}
\cline { 3 - 3 }$T_{E \mathbf{6}}$ & $\bigcirc$ & $\varnothing$ & 0.1 \\
\cline { 2 - 3 }
\end{tabular} & {$\left[\begin{array}{cc}\mathbf{0} & \mathbf{0} \\
d_{y} & \delta_{y} \\
d_{z} & \delta_{z}\end{array}\right]$} & $\begin{aligned}-\frac{\phi t}{\mathbf{2}} & \leq d_{y} \leq \frac{\phi t}{\mathbf{2}} ;-\frac{\phi t}{\mathbf{2}} \leq d_{z} \leq \frac{\phi t}{\mathbf{2}} \\
-\frac{\phi t}{L} & \leq \delta_{y} \leq \frac{\phi t}{L} ;-\frac{\phi t}{L} \leq \delta_{z} \leq \frac{\phi t}{L} \\
\delta_{y}{ }^{2}+\delta_{z}^{2} & \leq\left(\frac{\phi t}{\mathbf{2}}\right)^{2}\end{aligned}$ & $\begin{array}{l}\text { Triangular } \\
\text { distribution }\end{array}$ \\
\hline
\end{tabular}

Calculation and Simulation Cumulative Tolerance. Through calculating each tolerance value related to the base coordinate system, centering precision from the axis of the centering pin to the surface in the base coordinate system can be acquired in the $\mathrm{Z}$ direction as shown: 
Table 2 Tolerances of functional elements of centering pin mechanism

\begin{tabular}{cccccccc}
\hline & $T_{E 1}$ & $T_{E 2}$ & $T_{E 3}$ & $T_{E 4}$ & $T_{E 5}$ & $T_{E \mathbf{6}}$ & $T_{A R}$ \\
\hline Medium deviation & 0 & 0 & 0 & 0.0165 & -0.0115 & 0 & 0.005 \\
Standard deviation & $\left(\begin{array}{c}+0.0125 \\
-0.0125\end{array}\right)$ & $\left(\begin{array}{c}+0.0125 \\
-0.0125\end{array}\right)$ & $\left(\begin{array}{c}+0.10 \\
-0.10\end{array}\right)$ & $\left(\begin{array}{c}+0.033 \\
0\end{array}\right)$ & $\left(\begin{array}{c}-0.007 \\
-0.016\end{array}\right)$ & $\left(\begin{array}{c}+0.1083 \\
-0.1083\end{array}\right)$ & $\left(\begin{array}{c}+0.1870 \\
-0.1770\end{array}\right)$ \\
contribution & $4.91 \%$ & $4.91 \%$ & $39.34 \%$ & $6.43 \%$ & $1.91 \%$ & $42.5 \%$ & $100 \%$ \\
\hline
\end{tabular}

Based on the method above, the small displacement torsor matrix can be solved, which define the relationship between each functional element to the base coordinate system. Select the corresponding tolerance components which direction was consistent with that of TAR. And then the value of TAR can be gotten by sum. Finally calculate the contributions of partial components to closed component respectably. Consequently, the contributions can be helpful to adjust the accuracy of the functional elements to meet the functional requirements.

\section{Conclusion}

A tolerance analysis method has been used by robot kinematics theory in this paper. Consider geometric tolerance as a partial component in tolerance chain, along with size tolerance. Through a numerical and graphical approach, the percentage contribution has been calculated to guide the designer into choosing the proper tolerances. This method can tell the designer how to modify the most critical tolerance so as to respect the functional requirement imposed.

\section{References}

[1] Desrochers, A.; Ghie, W.; Laperrière, L. : Application of a unified Jacobian-Torsor model for tolerance analysis; In: Journal of Computing and Information Science in Engineering; v3;n1; pp. 2-14; 2003.

[2] Ghie, W.;Desrochers, A.; Laperrière, L: Re-Design of Mechanical Assemblies using the Unified Jacobian-Torsor Model for Tolerance Analysis; In: Models for Computer Aided Tolerancing in Design and Manufacturing, 95-104. 2007.

[3] E. E. Lin ;d H.-C. Zhang, Theoretical Tolerance Stackup Analysis Based on Tolerance Zone Analysis, Int J Adv Manuf Technol, 17: 257-262. 2001.

[4] Desrochers, A.; riviere. A: A Mxtrix Approach to the Representation of Tolerance Zones and Clearances, Int J Adv Manuf Technol (1997) 13:630-636; 1997.

[5] Requicha, A. A. G.; Toward a Theory of Geometrical Tolerancing, In: International Journal Robotics Research; v2,n4, pp. 45-60; 1983.

[6] Zhang Wu, Sensitive Factor for Position Tolerance, In: Research in Engineering Design (1997) 9:228-234 